\title{
MODELOS MATEMÁTICOS PARA O CRESCIMENTO DA POPULAÇÃO DO ESTADO DE SÃO PAULO E A EXPLORAÇÃO DE DIFERENTES TAXAS DE CRESCIMENTO
}

\section{Mathematical models for São Paulo population growth and the exploration of different growth rates}

\author{
Maria Beatriz Ferreira Leite ${ }^{1}$ \\ Gabriella Helena Jorge da Silva ${ }^{2}$ \\ Livia Fernandes de Sousa ${ }^{3}$
}

\begin{abstract}
Resumo: A utilização de modelos matemáticos que descrevem a evolução temporal de populações (crescimento ou decréscimo) pode ser extremamente útil para a escolha de medidas preventivas e/ou de controle. São muitas as ferramentas matemáticas disponíveis, e uma escolha adequada depende tanto do tipo de informações disponíveis quanto dos objetivos almejados. Buscando explorar conteúdos matemáticos do Ensino Médio, neste trabalho são apresentados alguns modelos matemáticos que descrevem o crescimento da população do Estado de São Paulo, a partir da utilização de diferentes funções. Diferentes taxas de crescimento são exploradas, e as características e hipóteses básicas de cada modelo proposto são apresentadas. Uma comparação quantitativa e qualitativa é feita para validar os modelos obtidos.
\end{abstract}

Palavras-chave: Modelos matemáticos. Dinâmica populacional. Taxas de crescimento.

\begin{abstract}
The use of mathematical models which describe the evolution of populations through the time (increase or decrease) can be very useful for making choices in preventing or controlling growth. There are so many mathematical tools available and an appropriate choice depends not only on the information but also on the expected goals as well. Aiming to explore mathematical ideas in high school, in this work some models are presented to describe the increase of São Paulo State's population, using different functions. Different growth rates are explored and the basic features and hypotheses of each proposed model are presented. A quantitative and qualitative comparison is made to validate the obtained models.
\end{abstract}

Keywords: Mathematical models. Dynamics of population. Growth rates.

\footnotetext{
${ }^{1}$ Matemática, doutorado em Matemática Aplicada. Docente, Faculdade de Matemática, Pontifícia Universidade Católica de Campinas (PUC-Campinas).Campinas, SP, Brasil. < bialeite@puc-campinas.edu.br>

${ }^{2}$ Discente, curso de Licenciatura em Matemática, PUC-Campinas. Campinas, SP, Brasil.<gabriellahjs@gmail.com>

${ }_{3}^{3}$ Discente, curso de Análise de Sistemas. PUC-Campinas. Campinas, SP, Brasil. <liviafsousa@gmail.com>
}

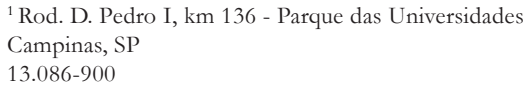




\section{Introdução}

Neste artigo são apresentados modelos matemáticos que resultaram do desenvolvimento de um trabalho realizado no âmbito da iniciação científica, envolvendo alunas de diferentes áreas de conhecimento (dos cursos de Licenciatura em Matemática e Análise de Sistemas). A proposta era trabalhar a contextualização de conteúdos matemáticos a partir da aplicação da modelagem matemática aliada à utilização de recursos computacionais. No caso da aluna do curso de Licenciatura em Matemática, enfatizou-se a modelagem matemática como estratégia no processo de ensino e aprendizagem. Vale notar que diferentes abordagens para a utilização da modelagem como instrumento pedagógico são apresentadas na literatura, como as perspectivas pragmática e científica (KAISER, 1995) e sociocrítica (BARBOSA, 2003). A diferença entre cada uma dessas perspectivas é a forma como o processo de modelagem é conduzido. Em linhas gerais, nas perspectivas pragmática e científica, maior ênfase é dada à construção dos modelos e aos conteúdos matemáticos envolvidos. Por outro lado, na perspectiva sociocrítica, exploram-se mais as discussões que podem decorrer da análise do problema abordado. No escopo deste trabalho foi enfatizada a construção de modelos matemáticos e a exploração de conteúdos do Ensino Médio, evidenciando, assim, a abordagem pragmática e científica. A principal razão do enfoque assumido foi apresentar a modelagem matemática como uma estratégia para um ensino de Matemática mais significativo, por meio da contextualização de conteúdos matemáticos na resolução e análise de problemas. Para a aluna do Curso de Análise de Sistemas, a ênfase foi dada na aplicação de recursos computacionais na resolução e simulação de modelos. A integração das duas áreas proporcionou um enriquecimento significativo do plano de trabalho de ambas as alunas, ampliando o caráter específico dos planos de trabalho e viabilizando a prática da interdisciplinaridade.

De maneira geral, os modelos matemáticos podem ser elaborados a partir da utilização de ferramentas matemáticas distintas, dependendo das características dos problemas, do tipo de dados disponíveis, tais como: equações contínuas ou discretas, matrizes, conjuntos fuzzy, entre outras (BASSANEZI, 2002; EDELSTEIN-KESHET, 1988; BARROS; BASSANEZI, 2006). Exemplos de modelos matemáticos aplicados a problemas ambientais que utilizam conteúdos matemáticos distintos são apresentados em Leite, Ferreira e Scrich (2009).

Buscando aplicar a modelagem matemáticae trabalhar com conteúdos matemáticos compatíveis com o currículo do Ensino Médio, o tema abordado neste artigo foi Dinâmica Populacional. A descrição do tamanho de populações ao longo do tempo, por meio de modelos matemáticos, pode ser bastante útil não apenas para prever a evolução populacional como, também, para possibilitar a análise de fatores que contribuem na sua dinâmica. A partir da análise e simulação de modelos matemáticos, é possível avaliar, por exemplo, quando uma determinada população corre risco de se extinguir, ou estimar o tempo necessário para que atinja um determinado nível e, desta forma, planejar ações adequadas. Contudo, nesta análise, uma população raramente pode ser considerada isolada de um biossistema, no qual várias populações se relacionam de diferentes formas. O estudo da dinâmica populacional também possibilita perceber como ocorreu o processo de evolução na própria construção dos modelos matemáticos, a partir de diferentes hipóteses e suposições incorporadas.

Dentro deste vasto e abrangente assunto, escolheu-se modelar o crescimento da população do estado de São Paulo. Uma vez que o objetivo era contextualizar conteúdos mate- 
máticos do Ensino Médio, o crescimento desta população foi modelado por três tipos de funções: linear, exponencial e logística. As taxas de crescimento foram calculadas considerando-se as características particulares e hipóteses básicas de cada tipo de modelo. Os gráficos e simulações foram feitos através dos softwares Winplot e Excel, também visando recursos acessíveis para utilização dos mesmos em sala de aula.

\section{A modelagem matemática e a elaboração dos modelos matemáticos}

O desenvolvimento do trabalho das alunas envolvidas baseou-se, essencialmente, na modelagem matemática como metodologia. Inicialmente, ambas estudaram o processo de modelagem, suas etapas, características e exemplos de aplicações. De modo geral, na modelagem matemática, após a definição do tema, procede-se a coleta de dados. Quando não se tem um problema a priori, a investigação sobre o tema possibilita que seja identificado e definido um problema a ser abordado. $\mathrm{Na}$ fase denominada abstração, as hipóteses são formuladas, e assumidas as simplificações que fundamentam a elaboração do modelo matemático. A linha tracejada indica a retomada do processo, a partir de novos dados e/ou modificação das hipóteses. A Figura 1 sintetiza as etapas do processo.

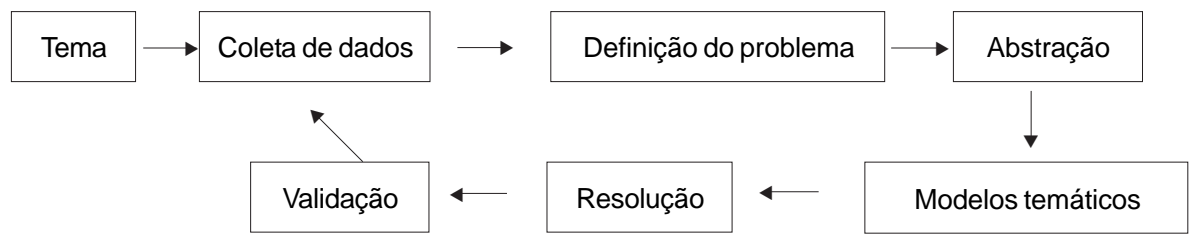

Figura 1. Etapas do processo de modelagem matemática.

Fonte: Leite, Silva e Sousa, dados da pesquisa.

Dentre as diversas possibilidades que se apresentam no amplo e abrangente tema escolhido, Dinâmica Populacional, optou-se pela análise do crescimento da população do Estado de São Paulo. Como a principal proposta do trabalho de iniciação científica era elaborar modelos matemáticos para o Ensino Médio, visando à contextualização de conteúdos e uma aprendizagem mais significativa, optou-se por explorar o conteúdo de funções, usando diferentes formulações para descrever a evolução da população considerada. O principal objetivo na construção de diferentes modelos foi enfatizar a flexibilidade da modelagem matemática como estratégia de ensino, viabilizando a modificação de modelos por meio da incorporação de novas hipóteses. 
Leite M. B. F.; Silva, G. H. J.; Sousa, L. F.

Os dados utilizados foram coletados no site da Fundação Sistema Estaduais de Análise de Dados (SEADE, 2008). O ano de 1994 foi identificado como instante inicial $(t=0) \mathrm{e}$, dessa forma, o ano de 2003 corresponde a $t=9$. Os valores da população do Estado de São Paulo nesse período estão na Tabela 1 e representados na Figura 2.

Tabela 1. População do Estado de São Paulo.

\begin{tabular}{ccc} 
Ano & Tempo & Número de habitantes \\
\hline 1994 & 0 & 33.162 .862 \\
1995 & 1 & 33.486 .396 \\
1996 & 2 & 34.074 .644 \\
1997 & 3 & 34.581 .838 \\
1998 & 4 & 35.124 .979 \\
1999 & 5 & 36.276 .632 \\
2000 & 6 & 36.909 .200 \\
2001 & 7 & 37.542 .521 \\
2002 & 8 & 38.123 .695 \\
2003 & 9 & 38.718 .301 \\
\hline
\end{tabular}

Fonte: Fundação Sistema Estadual de Análise de Dados (SEADE).

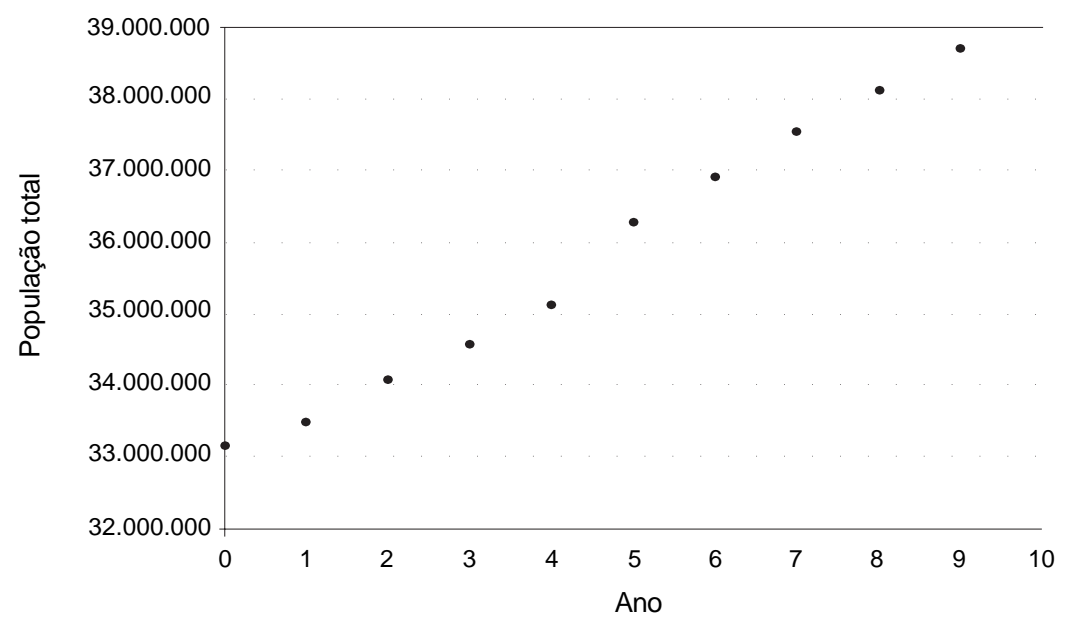

Figura 2. População do Estado de São Paulo de 1994 a 2003.

Fonte: Leite, Silva e Sousa, dados da pesquisa.

A seguir, são apresentados os modelos matemáticos elaborados a partir da utilização de três tipos de funções: linear, exponencial e logística. Buscou-se evidenciar como, a partir da incorporação de diferentes suposições e hipóteses, a formulação dos modelos matemáticos pode evoluir. Vale observar que essa prática não é tão simples e, em um primeiro contato com 
a modelagem matemática, muitas foram as dúvidas apresentadas pelas alunas, tanto na própria contextualização dos conteúdos matemáticos como, também, na elaboração dos modelos matemáticos.

\section{O modelo linear}

No modelo linear, a suposição básica é que a variação do número total de indivíduos é constante. Em outras palavras, admite-se que o acréscimo (ou decréscimo) da quantidade de indivíduos por unidade de tempo não varia com o tempo. Além disso, supõe-se que não há restrições nem tampouco limitações para o crescimento (ou decrescimento), e, dessa forma, a população cresce (ou decresce) ilimitadamente.

A expressão geral que representa o número de indivíduos $N$ em função do tempo $t$ admitindo-se crescimento linear é dada por $\mathrm{N}=\mathrm{rt}+\mathrm{N}_{0}$, onde $r$ é a taxa de crescimento, té o tempo e $N_{0}$ é a população inicial.

Usando os dados apresentados na Tabela 1, consideramos $N(0)=33162862$, que corresponde à população total do Estado de São Paulo no ano de 1994. A estimativa da taxa de crescimento

$$
\mathrm{r}=\frac{\Delta N}{\Delta t}
$$

foi feita a partir da média da variação anual do número de indivíduos $(\Delta t=1)$, conforme valores exibidos na Tabela 2 .

\begin{tabular}{|c|c|c|}
\hline Tempo & População Total & $\begin{array}{l}\text { Taxa de crescimento } \\
\text { (número de habitantes/ano) }\end{array}$ \\
\hline 0 & 33.162 .862 & \\
\hline 1 & 33.486 .396 & 323.534 \\
\hline 2 & 34.074 .644 & 588.248 \\
\hline 3 & 34.581 .838 & 507.194 \\
\hline 4 & 35.124 .979 & 543.141 \\
\hline 5 & 36.276 .632 & 1.151 .653 \\
\hline 6 & 36.909 .200 & 632.568 \\
\hline 7 & 37.542 .521 & 633.321 \\
\hline 8 & 38.123 .695 & 581.174 \\
\hline 9 & 38.718 .301 & 594.606 \\
\hline
\end{tabular}

Fonte: Leite, Silva e Sousa, dados da pesquisa.

O valor médio obtido foi de $\mathrm{r}=\frac{5.555 .439}{9}=617.271$

A Figura 3 mostra o gráfico da função de primeiro grau que representa o Modelo Linear e os pontos originais apresentados na Tabela 1. 
Leite M. B. F.; Silva, G. H. J.; Sousa, L. F.

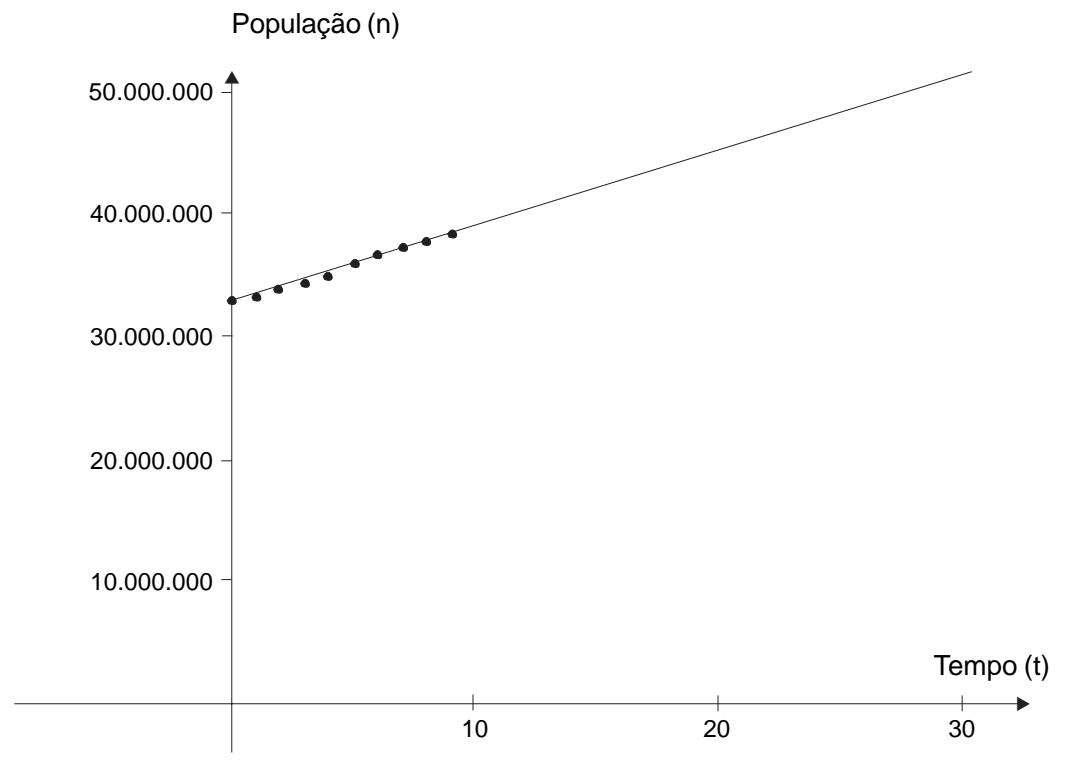

Figura 3. Modelo linear $\mathrm{N}=617.217 t+33.162 .862$ para a população do Estado de São Paulo.

Fonte: Leite, Silva e Sousa, dados da pesquisa.

Vale observar que o mesmo resultado poderia ser obtido por meio do estudo de uma progressão aritmética (PA), possibilitando a exploração de outros conteúdos matemáticos equivalentes.

Entretanto, na modelagem de crescimento populacional, nem sempre é razoável supor que a quantidade de indivíduos acrescida na população é constante. Esta suposição pode ser válida para um determinado período de tempo ou para alguma população em particular. Admitindo que o número de indivíduos que é acrescido depende, por exemplo, do tamanho da própria população, o modelo linear não é mais adequado. A seguir apresentamos outras possibilidades para modelar o crescimento populacional.

\section{O modelo exponencial}

No modelo exponencial, a hipótese básica é que a população cresce sem qualquer restrição, não admitindo fatores que regulam seu crescimento, tais como: epidemias, guerras, fome, entre outros. Supõe-se que o número total de indivíduos varia com o tempo, dependendo da quantidade presente em cada instante. Admitindo-se que a taxa de crescimento é contínua, o modelo exponencial é descrito pela função $N=N_{0} e^{r t}$, onde $N_{0}$ é a população inicial, $r$ é a taxa de crescimento e $t$ é o tempo. 
A estimativa da taxa de crescimento foi feita considerando-se o crescimento relativo em cada ano, isto é, comparando-se o acréscimo do número de indivíduos em relação à população anterior. Dois procedimentos distintos foram adotados para a obtenção da taxa de crescimento $r$. uma média das taxas relativas anuais e a taxa relativa total do período, conforme os casos a e b descritos a seguir.

a) Média das taxas

A estimativa da taxa de crescimento, neste caso, foi feita considerando-se o crescimento relativo em cada ano, isto é, comparando-se o acréscimo do número de indivíduos em relação à população anterior.

Neste caso foram calculados os acréscimos relativos anuais e a taxa foi obtida considerando-se a média dos acréscimos relativos no período, conforme valores mostrados na Tabela 3 .

Tabela 3. Acréscimos relativos anuais do número de indivíduos no Estado de São Paulo.

\begin{tabular}{lcc}
\hline Tempo & População Total & Crescimento relativo \\
\hline 0 & 33.162 .862 & 0,0098 \\
1 & 33.486 .396 & 0,0176 \\
2 & 34.074 .644 & 0,0149 \\
3 & 34.581 .838 & 0,0157 \\
4 & 35.124 .979 & 0,0328 \\
5 & 36.276 .632 & 0,0174 \\
6 & 36.909 .200 & 0,0172 \\
7 & 37.542 .521 & 0,0155 \\
8 & 38.123 .695 & 0,0156 \\
9 & 38.718 .301 & 0,1564 \\
Total & & \\
\hline
\end{tabular}

Fonte: Leite, Silva e Sousa, dados da pesquisa.

Assim, obtemos $r=\frac{0,1564}{9}=0,0174$.

b) Taxa total

Neste caso foi obtido o acréscimo relativo total do período,

$$
\frac{\Delta N}{N}=\frac{N_{\text {final }}-N_{\text {inicial }}}{N_{\text {nicial }}}=\frac{38.718 .301-33.162 .862}{33.162 .862}=0,1675
$$

e a taxa de crescimento resultante foi de $\mathrm{r}=\frac{0,1675}{9} \rightarrow r=0,0186$.

A Figura 4 mostra o gráfico da função exponencial e os pontos originais apresentados na Tabela 1. 
Leite M. B. F.; Silva, G. H. J.; Sousa, L. F.

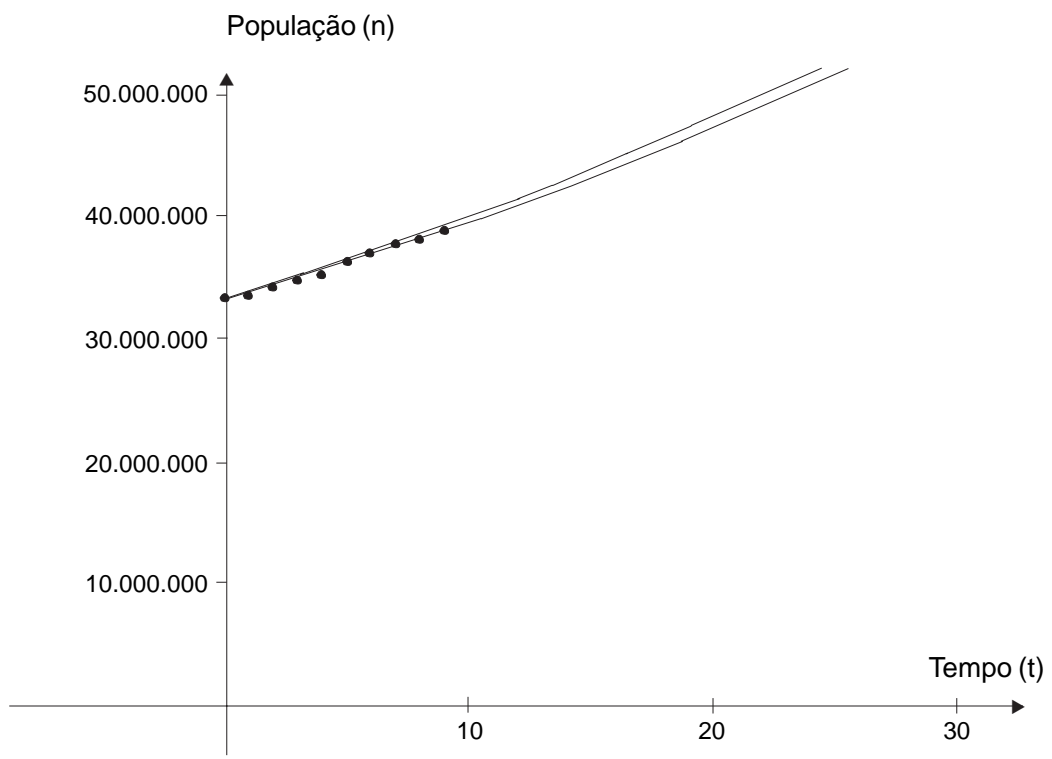

Figura 4. Modelos exponenciais para os casos (a) e (b) descritos acima.

Fonte: Leite, Silva e Sousa, dados da pesquisa.

É importante ressaltar que foi admitido crescimento contínuo e, por isso, a função exponencial considera a base natural $e$. Entretanto, também é possível trabalhar, por exemplo, com o crescimento anual (ou mensal) e, com isso, explorar diferentes bases para as funções exponenciais do tipo $N=N_{0} a^{r t}$. Novamente, vale observar que é possível também, a partir das mesmas hipóteses, explorar o estudo de uma progressão geométrica (PG).

Como o modelo exponencial não prevê qualquer tipo de inibição para o crescimento populacional, o mesmo pode não ser adequado para representar o que ocorre em muitas populações em longo prazo. Em muitas situações, é razoável considerar que o crescimento da população é inibido por fatores como: falta de espaço, escassez de alimentação, etc. Em outras palavras, pode-se supor que a taxa de crescimento populacional diminui com o crescimento da população, e que existe uma capacidade máxima sustentável para essa população. Vários modelos matemáticos podem representar essa hipótese, como, por exemplo, o modelo Logístico, que é descrito a seguir.

\section{O modelo logístico}

A formulação do modelo logístico supõe que a população sofre inibições naturais no seu crescimento. Em outras palavras, admite-se que a taxa de crescimento decresce com a população. Para pequenas quantidades de indivíduos, quando não há escassez de recursos, o 
modelo comporta-se de forma análoga ao modelo exponencial. À medida que a população cresce, a competição entre os indivíduos faz com que a taxa de crescimento diminua, embora o número de indivíduos continue crescendo. Neste modelo, admite-se que há um nível populacional máximo, que descreve a capacidade suporte do meio. Uma vez atingido este nível, a população seria estável. A função que descreve este modelo é dada por

$$
\mathrm{N}=\frac{K \cdot N_{0}}{N_{0}+\left(K-N_{0}\right) \cdot e^{-r t}},
$$

onde $N$ é o número de indivíduos no instante t, $K$ representa o valor-limite da população, $N_{0}$ é a população inicial, $r$ é a taxa de crescimento, e $t$ é o tempo.

Para estimar qual seria esse valor-limite para a população do Estado de São Paulo, utilizou-se o método de Ford-Walford (BASSANEZI, 2002, p. 72). Para que este método forneça uma boa estimativa do valor de $K$, é necessário que sejam conhecidos níveis populacionais, não do início do processo (onde o modelo funciona como o modelo exponencial), mas valores populacionais do período no qual a taxa de crescimento já começou a diminuir. Observando a Tabela 3, verifica-se que isto ocorre a partir de 1999 ( $t=5)$.

Basicamente, este método supõe que, uma vez em equilíbrio, a população não varia mais, isto é $\mathrm{N}_{t+1}=\mathrm{N}_{t}$. Assim, a estimativa deste valor-limite pode ser obtida relacionando os valores das populações nos instantes $t$ e $t+1$. A partir dos pontos obtidos, ajusta-se uma reta que descreve como as populações consecutivas estão relacionadas $N_{t+1}=f\left(N_{t}\right)=a N_{t}+b$, conforme mostrado na Figura 5. Para encontrar o valor de equilíbrio, basta determinar a intersecção desta reta com a bissetriz, uma vez que estamos supondo que, no valor-limite, a população não varia, isto é $\mathrm{N}_{t+1}=\mathrm{N}_{t}$.

Assim, nas estimativas dos valores de $k$ e $r$ para este modelo, consideramos os valores populacionais de 1999 a 2003 (ver Tabela 4).

Tabela 4. Valores utilizados no
método de Ford-Walford
\begin{tabular}{ccc}
\hline $\boldsymbol{t}$ & $\boldsymbol{N}_{\boldsymbol{t}}$ & $\boldsymbol{N}_{\boldsymbol{t + 1}}$ \\
\hline 5 & 36.276 .632 & 36.909 .200 \\
6 & 36.909 .200 & 37.542 .521 \\
7 & 37.542 .521 & 38.123 .695 \\
8 & 38.123 .695 & 38.718 .301 \\
\hline
\end{tabular}

Fonte: Leite, Silva e Sousa, dados da pesquisa.

Fazendo a intersecção desta reta com a bissetriz, obtemos o valor-limite da população $K=2000000 /(1-0,9729)=73800738$.

A estimativa da taxa de crescimento $r$ foi feita com base nos valores do SEADE (2008) e na expressão do próprio modelo. Isolando-se o valor de $r$ no modelo, obtemos

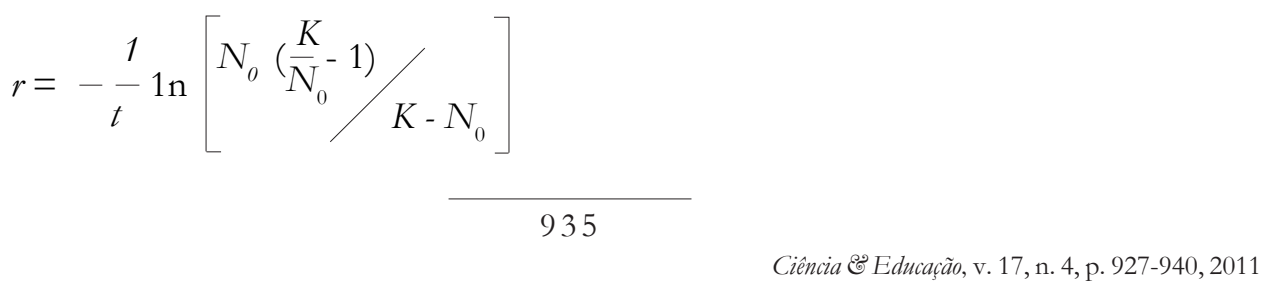


Leite M. B. F.; Silva, G. H. J.; Sousa, L. F.

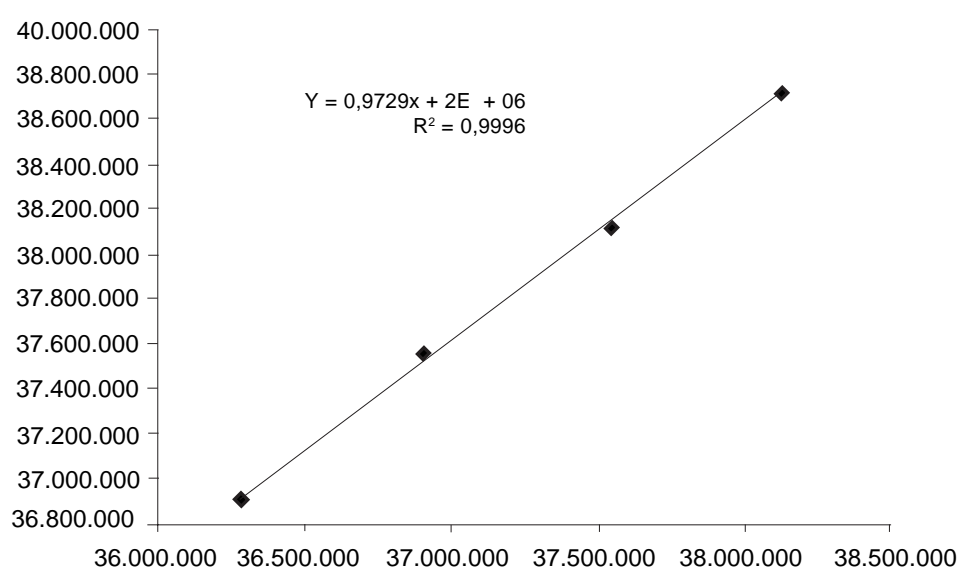

Figura 5. Reta ajustada para $\mathrm{N}_{t+1}=\mathrm{f}\left(\mathrm{N}_{t}\right)$.

Fonte: Leite, Silva e Sousa, dados da pesquisa;

A Tabela 5 apresenta os valores obtidos para $r$ a cada ano, a partir de 1999.

Tabela 5. Estimativa da taxa de crescimento $r$ para o modelo logístico

\begin{tabular}{ccr}
\hline $\boldsymbol{t}$ & $\boldsymbol{N}_{\boldsymbol{t}}$ & \multicolumn{1}{c}{$\boldsymbol{r}$} \\
\hline 5 & 36.276 .632 & 0,033892027 \\
6 & 36.909 .200 & 0,03395809 \\
7 & 37.542 .521 & 0,034011151 \\
8 & 38.123 .695 & 0,03369981 \\
9 & 38.718 .301 & 0,033542406 \\
\hline
\end{tabular}

Fonte: Leite, Silva e Sousa, dados da pesquisa.

O valor médio obtido para o período considerado foi de 0,033821 . Nos cálculos e simulações, o valor utilizado foi de 0,0338 .

A Figura 6 mostra o gráfico da função obtida no Modelo Logístico e os pontos originais apresentados na Tabela 1. 
Modelos matemáticos para o crescimento ...

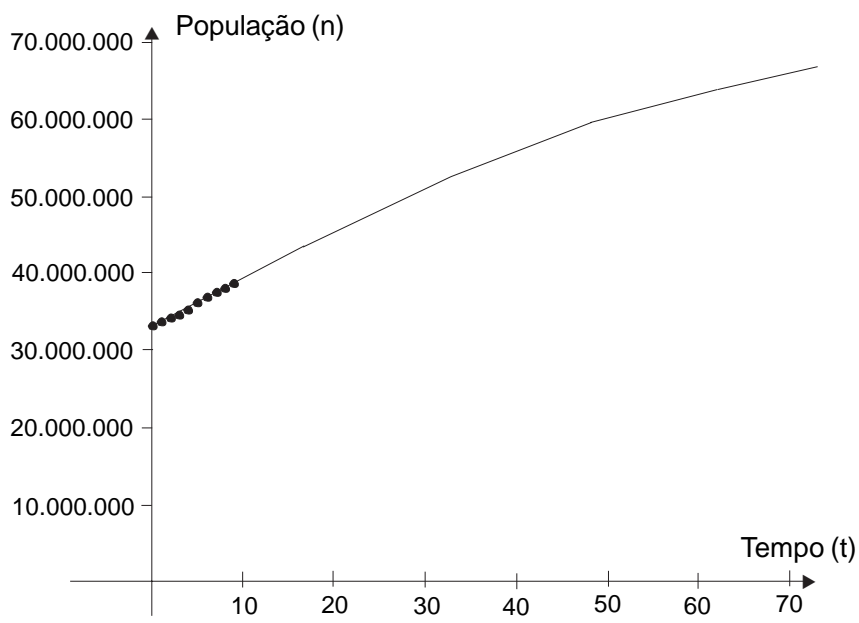
Figura 6. Modelo Logístico $\mathrm{N}=\frac{(73.800 .738)(33.162 .862)}{33.162 .862+40.637 .876 e^{-0,0338 t}}$ para a população do Estado de São
Paulo.

Fonte: Leite, Silva e Sousa, dados da pesquisa.

\section{Comparando os modelos}

Uma comparação básica entre os três modelos propostos foi feita a partir do cálculo da diferença entre os valores originais e os valores estimados. Os resultados estão na Tabela 6.

\begin{tabular}{|c|c|c|c|}
\hline$t$ & $N_{\text {OBS }}-N_{\text {LINEAR }}$ & $N_{\text {OBS }}-N_{\text {EXP }}$ & $N_{O B S}-N_{L O G}$ \\
\hline 0 & 0 & 0 & 0 \\
\hline 1 & 293.737 & 258.549 & 294.684 \\
\hline 2 & 322.760 & 262.601 & 326.423 \\
\hline 3 & 432.837 & 358.104 & 440.636 \\
\hline 4 & 506.967 & 428.238 & 519.972 \\
\hline 5 & 27.415 & 99.376 & 8.487 \\
\hline 6 & 42.712 & 96.951 & 17.501 \\
\hline 7 & 58.762 & 84.134 & 27.262 \\
\hline 8 & 22.665 & 7.829 & 14.772 \\
\hline 9 & 0 & 66.585 & 42.668 \\
\hline$\Sigma$ & 1.707 .855 & 1.662 .367 & 1.692 .405 \\
\hline
\end{tabular}

Fonte: Leite, Silva e Sousa, dados da pesquisa. 
Nota-se que, para os valores no período de 1994 a 2003, o modelo que mais se aproxima dos dados observados é o exponencial (a soma das diferenças é a menor). Entretanto, sabe-se que o crescimento ilimitado não é razoável em longo prazo, o que faria com que fosse descartado tanto o modelo linear quanto o exponencial para projeções populacionais futuras. Além disso, observa-se que o erro do modelo logístico proposto se aproxima do erro do modelo exponencial e contempla a questão da população-limite.

Outra forma de validar os modelos propostos é comparar o valor obtido por meio das funções com valores reais. Por exemplo, para o ano de 2007 ( $t=13$ ), o valor fornecido pelo Instituto Brasileiro de Geografia e Estatística - IBGE (2009) foi de 39.827.570. Na Tabela 7, as diferenças das estimativas de cada modelo são apresentadas.

\begin{tabular}{|c|c|c|c|}
\hline & Linear & Exponencial & Logístico \\
\hline$N(13)$ & 41.187 .385 & 41.580 .472 & 41.236 .885 \\
\hline$N_{I B G E}-N(t)$ & 1.359 .815 & 1.752 .905 & 1.409 .315 \\
\hline
\end{tabular}

Fonte: Leite, Silva e Sousa, dados da pesquisa.

Para este valor do tempo $t$, o erro cometido através do modelo linear novamente é o menor e está próximo do modelo logístico, que contempla a hipótese de a população ter uma capacidade-limite. Para projeções em curto prazo, tanto o modelo linear como o exponencial são adequados, mas, pelo fato de admitirem um crescimento ilimitado, espera-se que, à medida que o tempo passe, a estimativa através do modelo logístico seja mais razoável, e o erro cometido menor.

Vale observar ainda que muitos outros modelos populacionais podem ser elaborados a partir da incorporação de novas suposições. O objetivo aqui foi - sem avançar em conteúdos matemáticos do Ensino Superior - aplicar o estudo de funções e explorar o conceito de taxas a partir de diferentes hipóteses.

\section{Comentários finais}

A elaboração dos modelos matemáticos apresentados proporcionou, além do aprofundamento dos conhecimentos específicos das alunas envolvidas, a vivência do trabalho interdisciplinar e em equipe. Neste sentido, vale ressaltar como a modelagem matemática, como estratégia pedagógica (por ser naturalmente interdisciplinar), proporciona e viabiliza a prática da interdisciplinaridade e facilita a contextualização de conteúdos matemáticos. Cabe ainda observar que, como trabalho de pesquisa no âmbito da Iniciação Científica, foi utilizada a metodologia da modelagem matemática, proporcionando a experimentação de todo o pro- 
cesso, desde a coleta de dados até a análise e validação dos modelos obtidos. Particularmente para o modelo logístico, vale enfatizar a importância das simulações (através da opção de animações) realizadas no Winplot. Só a partir destas simulações foi possível inferir sobre o valor ideal da taxa de crescimento para este modelo (que se mostrava em torno de 0,0335), e buscar uma forma de obtê-la a partir dos dados experimentais. Além disso, foi possível encontrar uma justificativa matemática para isto de acordo com as suposições do modelo (que, no caso, foi considerar os dados referentes ao período no qual a taxa de crescimento começa a diminuir). Outro aspecto relevante do trabalho com a modelagem matemática é a possibilidade que ela traz para que sejam explorados e, até mesmo, construídos, mesmo que intuitivamente, importantes conceitos matemáticos. Por exemplo, apesar de este trabalho não abordar conteúdos do Ensino Superior, o estudo das taxas de crescimento possibilita a introdução do conceito de derivada, a partir da análise da variação do tamanho da população.

Evidencia-se, também, como a construção de modelos matemáticos possibilita a integração entre pesquisa e ensino, uma vez que, na modelagem matemática, a prática da investigação é indispensável e foi amplamente explorada no trabalho desenvolvido com as alunas, frente às dúvidas e questionamentos apresentados. De fato, para Biembengut (2004, p. 23):

Como é essencialmente um método de pesquisa, no Ensino, a modelagem matemática pode tornar-se caminho para despertar no aluno interesse por assuntos de matemática e, também, de alguma área da ciência que ainda desconheça ao mesmo tempo em que ele aprende a arte de modelar, matematicamente.

A modificação dos modelos a partir da inclusão de novas suposições e hipóteses, compreendendo seus significados e suas implicações, caracteriza um importante aspecto da modelagem matemática que, no escopo deste trabalho, foi bastante explorado e indiscutivelmente colaborou para que ricas e produtivas discussões surgissem. Com a incorporação de diferentes características, a modificação dos modelos matemáticos foi também essencial para propiciar uma visão dinâmica do processo de ensino, na qual os conteúdos matemáticos não são vistos como prontos e acabados, mas como ferramentas de análise, úteis e flexíveis, que podem ser adaptadas e aplicadas em diferentes contextos. 
Leite M. B. F.; Silva, G. H. J.; Sousa, L. F.

\section{Referências}

BARBOSA, J. What is mathematical modelling? In: LAMON, S. J.; PARKER, W. A.; HOUSTON, S. K. (Eds.). Mathematical modelling: a way of life. Chichester: Horwood, 2003. p. 227-234. (ICTMA, 11).

BARROS, L. C.; BASSANEZI, R. C. Tópicos de lógica fuzzy e biomatemática. Campinas: IMECC, 2006. (Textos Didáticos, 5).

BASSANEZI, R. C. Ensino-aprendizagem com modelagem matemática. São Paulo: Contexto, 2002.

BIEMBENGUT, M. S. Modelagem matemática \& implicações no ensino-aprendizagem de matemática. 2. ed. Blumenau: Edifurb, 2004.

EDELSTEIN-KESHET, L. Mathematical models in biology. New York: Random House, 1988.

INSTITUTO BRASILEIRO DE GEOGRAFIA E ESTATÍSTICA. Contagem da população 2007. Disponível em: <www.ibge.gov.br/home/estatistica/populacao/ contagem2007>. Acesso em: 25 jun. 2009.

KAISER, G. Results from a comparative empirical study in England and Germany on the learning of mathematics in context. In: SLOYER, C.; BLUM, W.; HUNTLEY, I. (Eds.). Advances and perspectives in the teaching of mathematical modeling and applications. Yorklyn: Water Street Mathematics, 1995. p. 83-95.

LEITE, M. B. F.; FERREIRA, D. H. L.; SCRICH, C. R. Explorando conteúdos matemáticos a partir de temas ambientais. Ciência \& Educação, Bauru, v. 15, n. 1, p. 129-138, 2009.

SÃO PAULO (ESTADO). Fundação Sistema Estadual de Análise de Dados. Disponível em: <http://www.seade.gov.br/>. Acesso em: 10 nov. 2008.

Artigo recebido em 22/09/2010. Aceito em 26/03/2011. 